\title{
COMPLICAÇÕES PRECOCES NO TRATAMENTO ORTOPÉDICO DAS METÁSTASES ÓSSEAS
}

\author{
EARLY COMPLICATIONS IN THE ORTHOPEDIC TREATMENT \\ OF BONE METASTASES
}

Luiz Eduardo Moreira Teixeira', Ricardo Horta Miranda ${ }^{2}$, Daniel Ferreira Ghediniं3, Rafael Bazílio Aguilar ${ }^{3}$, Eduardo Nilo Vasconcelos Novais ${ }^{4}$, Guilherme Moreira de Abreu e Silva ${ }^{3}$, Ivana Duval Araújo ${ }^{5}$, Marco Antônio Percope de Andrade ${ }^{6}$

\section{RESUMO}

Objetivo: Avaliar as complicações precoces do tratamento ortopédico de lesões ósseas metastáticas e os fatores associados a essas complicações. Métodos: Foram avaliados retrospectivamente 64 pacientes submetidos a tratamento cirúrgico de metástases ósseas, analisando as complicações ocorridas no peroperatório e pós-operatório precoce e associando-as com a origem do tumor, o tipo de procedimento realizado, a necessidade de reposição de sangue no peroperatório, necessidade de novos procedimentos cirúrgicos e a mortalidade em decorrência das complicações. Resultados: Complicações precoces do tratamento foram observadas em 17 (26,6\%); seis (35,2\%) evoluíram para óbito em decorrência dessa complicação. De acordo com o tipo, em 15 $(23,8 \%)$ casos foram complicações cirúrgicas, em quatro $(6,3 \%)$, clínicas e três $(4,7 \%)$ pacientes apresentaram tanto complicações clínicas quanto cirúrgicas. Não houve diferença significativa na frequência das complicações ou mortalidade quando avaliados o tipo de reconstrução ou o local acometido. Os tumores de origem renal necessitaram de maior reposição sanguínea e tiveram maior frequência de complicações $(p=0,0001)$. Conclusão: As complicações ocorreram em $26,6 \%$. As complicações não estão associadas ao tipo de tratamento realizado, nem ao local acometido. Os tumores de origem renal apresentaram risco aumentado de hemorragia.

Descritores - Neoplasias ósseas; Oncologia; Fraturas patológicas; Complicações pós-operatórias; Cirurgia ortopédica

\begin{abstract}
Objective: To assess the early complications in the orthopedic treatment of metastatic bone lesions and the factors associated with these complications. Method: There were assessed, retrospectively, 64 patients that underwent surgical treatment for bone metastases, analyzing the complications that occurred in the pre-operative and early post-operative period and associating them with the tumor origin, type of procedure done, the need of blood reposition before the surgery, the need of new surgical procedures and the mortality due to the complications. Results: Early complications in the treatment were observed in $17(26.6 \%)$ patients, of which six (35.2\%) ended up dying due to these complications. Regarding the type, 15 (23.8\%) cases were due to surgical complications, four (6.3\%) clinical and three (4.7\%) patients showed clinical and surgical complications. There was no significant difference in the frequency of complications or mortality when assessed the type of reconstruction or affected region. The tumors with a renal origin needed more blood reposition and showed a bigger frequency of complications. Conclusion: The complications occurred in $26.6 \%$. The complications are not related to the kind of treatment performed or to the region affected. The renal origin tumors showed a higher risk of hemorrhage.
\end{abstract}

Keywords - Bone neoplasms; Oncology; Fractures, spontaneous; Postoperative complications; Orthopedic surgery

\footnotetext{
1 - Mestre em Medicina; Coordenador do Ambulatório de Oncologia Ortopédica do Hospital das Clínicas da Universidade Federal de Minas Gerais - UFMG.

2 - Coordenador do Serviço de Oncologia Ortopédica da Santa Casa de Belo Horizonte.

3 - Médico Assistente do Serviço de Ortopedia e Traumatologia do Hospital das Clínicas da Universidade Federal de Minas Gerais - UFMG.

4 - Mestre em Medicina; Médico Assistente do Serviço de Ortopedia e Traumatologia do Hospital das Clínicas da Universidade Federal de Minas Gerais - UFMG.

5 - Professora Associada Doutora do Departamento de Cirurgia da Faculdade de Medicina da Universidade Federal de Minas Gerais - UFMG.

6 - Professor Adjunto Doutor do Departamento do Aparelho Locomotor da Faculdade de Medicina da Universidade Federal de Minas Gerais.
}

Trabalho realizado no Hospital das Clínicas da Universidade Federal de Minas Gerais (UFMG) e na Santa Casa de Belo Horizonte, Minas Gerais.

Correspondência: Avenida do Contorno, 7.485 - 30110-120 - Belo Horizonte, MG. E-mail: luizmteixeira@yahoo.com.br 


\section{INTRODUÇÃO}

A doença óssea metastática é a neoplasia maligna mais frequente do esqueleto, sendo o osso o terceiro sítio mais comum de disseminação dos adenocarcinomas, precedido apenas pelo pulmão e fígado ${ }^{(1)}$. A predisposição do tecido ósseo às metástases é explicada pelo elevado fluxo sanguíneo sob baixa pressão nas áreas de medula vermelha e por ser a matriz óssea um terreno propício para implante de células tumorais ${ }^{(2)}$.

Os avanços na terapia adjuvante e neoadjuvante, em especial da hormonoterapia e quimioterapia, têm melhorado a sobrevida dos pacientes com câncer e aumentado a prevalência de casos convivendo com metástases ósseas (MO). Isso torna o controle das $\mathrm{MO}$ fator essencial na melhora da qualidade de vida, no controle da dor e na manutenção da independência do paciente ${ }^{(1,3)}$.

Apesar de a maioria dos casos de MO ser de tratamento clínico, o acompanhamento ortopédico é capaz de identificar precocemente lesões que comprometam a estabilidade mecânica do esqueleto, prevenindo ou tratando fraturas, controlando a dor e reduzindo a morbidade ${ }^{(3)}$. Além da prevenção, o tratamento ortopédico é capaz de restaurar a capacidade de deambulação em $94 \%$ dos pacientes com fratura patológica ${ }^{(2)}$.

As complicações desse tratamento são comuns e influenciadas pelas condições clínicas do paciente, estágio da doença e pelo procedimento cirúrgico. Podem ocorrer tanto no peroperatório, pós-operatório imediato ou pós-operatório tardio e incluem complicações clínicas e cirúrgicas ${ }^{(1-3)}$.

O objetivo deste estudo é avaliar as complicações precoces de pacientes submetidos ao tratamento ortopédico de lesões ósseas metastáticas e os fatores associados a essas complicações.

\section{MÉTODOS}

\section{Pacientes}

Foram revistos os prontuários de pacientes registrados no Serviço de Oncologia Ortopédica do Hospital das Clínicas da Universidade Federal de Minas Gerais (UFMG) e da Santa Casa de Belo Horizonte entre janeiro de 2002 e março de 2007, que foram submetidos a tratamento cirúrgico ortopédico de lesões ósseas secundárias a neoplasias malignas extraesqueléticas. $\mathrm{O}$ estudo foi aprovado pelo comitê de ética dos respectivos hospitais. Foram incluídos no estudo 64 de um total de 90 pacientes. A exclusão de 26 pacientes ocorreu por dados insuficientes no prontuário $(n=12)$ e por exclusão dos tumores de origem medular: mieloma múltiplo $(n=9)$, leucemias $(n=3)$ e linfomas $(n=2)$.

A média de idade foi de 56,63 $\pm 14,26$ anos (29 a 85 anos), sendo $23(35,9 \%)$ pacientes do sexo masculino e 41 do feminino $(64,1 \%)$. Doze $(18,8 \%)$ pacientes eram melanodermas, $24(37,5 \%)$ feodermas e $28(43,8 \%)$ leucodermas. A sobrevida média foi de $9,20 \pm 11,96$ meses (mediana de 6,6 meses).

Tratamento radioterápico prévio foi realizado em $27(42,1 \%)$ e quimioterápico em $56(87,5 \%)$ pacientes. O motivo do encaminhamento para tratamento ortopédico foi fratura em $42(65,6 \%)$ pacientes, dor em 20 $(31,2 \%)$ e alteração nos exames em assintomáticos em dois $(3,2 \%)$ casos.

\section{Tumores}

Todas as MO localizadas em membros, cintura pélvica e escapular e que necessitaram de tratamento cirúrgico foram incluídas no estudo. Foram excluídos os tumores ósseos primários e os de origem medular. Quanto ao órgão de origem, o tumor mais comum foi o de mama em $23(35,9 \%)$ (Tabela 1). Quanto ao tipo histológico, as MO foram por adenocarcinomas em 34 $(53,1 \%)$ casos, por carcinomas em $23(35,9 \%)$ pacientes, sarcomas em cinco $(7,8 \%)$ e em dois $(3,1 \%)$ o tipo celular foi indeterminado.

Tabela 1 - Frequência das metástases de acordo com o órgão de origem

\begin{tabular}{l|c|c|c}
\hline \multicolumn{1}{c|}{ ORIGEM } & PACIENTES $(\mathrm{n})$ & $\begin{array}{c}\text { FREQUÊNCIA } \\
\text { ABSOLUTA (\%) }\end{array}$ & $\begin{array}{c}\text { FREQUÊNCIA } \\
\text { ACUMULADA(\%) }\end{array}$ \\
\hline MAMA & 23 & 35,9 & 35,9 \\
\hline RENAL & 8 & 12,5 & 48,4 \\
\hline PRÓSTATA & 8 & 12,5 & 60,9 \\
\hline PULMÃO & 4 & 6,3 & 67,2 \\
\hline COLORRETAL & 4 & 6,3 & 73,4 \\
\hline TIREOIDE & 3 & 4,7 & 78,3 \\
\hline OUTROS & 12 & 18,8 & 96,9 \\
\hline DESCONHECIDO & 2 & 3,1 & 100 \\
\hline TOTAL & 64 & 100 & 100 \\
\hline
\end{tabular}

Fonte: SAME Hospital das Clínicas da UFMG, Santa Casa - BH.

A localização mais comum foi nos membros inferiores em $52(81,3 \%)$ pacientes, sete casos localizados na pelve $(10,9 \%)$ e cinco $(7,9 \%)$ nos membros superiores. As lesões eram solitárias em $22(34,3 \%)$ pacientes e em múltiplas localizações no esqueleto em $42(65,7 \%)$ (Tabela 2). 
Tabela 2 - Distribuição anatômica das lesões submetidas ao tratamento cirúrgico de metástases ósseas

\begin{tabular}{l|c|c|c}
\hline \multicolumn{1}{c|}{ LOCALIZAÇÃO } & PACIENTES $(\mathrm{n})$ & FREQUÊNCIA (\%) & $\begin{array}{c}\text { FREQUÊNCIA } \\
\text { ACUMULADA (\%) }\end{array}$ \\
\hline PELVE & 7 & 10,9 & 10,9 \\
\hline COLO DO FÊMUR & 7 & 10,9 & 21,9 \\
\hline TROCANTERIANA & 25 & 39,1 & 60,9 \\
\hline SUBTROCANTERIANA & 13 & 20,3 & 81,3 \\
\hline DIÁFISE FEMORAL & 4 & 6,3 & 87,5 \\
\hline FÊMUR DISTAL & 2 & 3,1 & 90,6 \\
\hline TÍBIA & 1 & 1,6 & 92,2 \\
\hline ÚMERO PROXIMAL & 3 & 4,7 & 96,9 \\
\hline DIÁFISE UMERAL & 1 & 1,6 & 98,4 \\
\hline ÚMERO DISTAL & 1 & 1,6 & 100,0 \\
\hline TOTAL & $\mathbf{6 4}$ & $\mathbf{1 0 0 , 0}$ & 100,0 \\
\hline
\end{tabular}

Fonte: SAME Hospital das Clínicas da UFMG, Santa Casa - BH

A MO foi identificada como manifestação inicial da neoplasia em $12(18,8 \%)$ pacientes, ao diagnóstico do tumor primário em cinco $(7,8 \%)$, durante o tratamento oncológico em $20(31,3 \%)$ e após o término do tratamento em $27(42,2 \%)$.

\section{Tratamento ortopédico}

As indicações para o tratamento cirúrgico ortopédico foram: fraturas patológicas em $43(67,2 \%)$ pacientes, fraturas iminentes em $17(26,6 \%)$ e ressecção tumoral simples com objetivo curativo em quatro $(5,3 \%)$.

$\mathrm{O}$ objetivo da cirurgia era a estabilização rígida da fratura estabelecida ou iminente, permitindo deambulação imediata do paciente quando possível ou a ressecção ampla da lesão com objetivo curativo nas lesões isoladas, sem outros órgãos acometidos, e que apresentavam bom prognóstico oncológico. Artroplastias (convencionais, hemiartroplastias e endopróteses) foram realizadas em $24(37,5 \%)$ pacientes, osteossínteses (placas e hastes intramedulares) em $36(56,3 \%)$ e em quatro $(6,3 \%)$ não foram feitas reconstruções (Tabela 3 ).

A média da concentração sanguínea de hemoglobina no pré-operatório foi de $10,2 \pm 1,9 \mathrm{mg} / \mathrm{dL}$ e a média de reposição de concentrado de hemácias no peroperatório foi de 1,48 $\pm 1,2$ unidades por paciente (variando de zero a seis unidades); apenas 17 (26,6\%) dos pacientes não necessitaram de reposição.

\section{Complicações}

Foram avaliadas todas as complicações ocorridas no peroperatório e no primeiro mês de pós-operatório, sendo separadas em clínicas e cirúrgicas. As complicações
Tabela 3 - Métodos de fixação e reconstrução utilizados no tratamento das metástases ósseas

\begin{tabular}{l|c|c|c}
\hline \multicolumn{1}{c|}{ TRATAMENTO } & PACIENTES (n) & FREQUÊNCIA (\%) & $\begin{array}{c}\text { FREQUÊNCIA } \\
\text { ACUMULADA (\%) }\end{array}$ \\
\hline PLACA + PMMA & 23 & 35,9 & 35,9 \\
\hline HIM + PMMA & 11 & 17,2 & 53,1 \\
\hline PRÓTESE TOTAL & 9 & 14,1 & 67,2 \\
\hline ENDOPRÓTESE & 13 & 20,3 & 87,5 \\
\hline HEMIARTROPLASTIA & 2 & 3,1 & 90,6 \\
\hline PLACA ISOLADA & 1 & 1,6 & 92,1 \\
\hline HIM ISOLADA & 1 & 1,6 & 93,7 \\
\hline RESSECÇÃO & 4 & 6,3 & 100,0 \\
\hline TOTAL & 64 & 100,0 & 100,0 \\
\hline Legenda: PMMA - polimetilmetacrilato, HIM - haste intramedular. \\
Fonte: SAME - Hospital das Clínicas da UFMG, Santa Casa - BH.
\end{tabular}

clínicas incluíam as consequências sistêmicas do tratamento e as cirúrgicas eram decorrentes do efeito local durante o procedimento operatório e nos primeiros 30 dias de pós-operatório. Foram analisadas as frequências das complicações, a mortalidade, a necessidade de reposição de sangue medida pelo número de unidades de concentrado de hemácias utilizadas no peroperatório e a necessidade de procedimentos cirúrgicos adicionais.

Esses dados foram correlacionados com os seguintes parâmetros: origem do tumor, o tipo de procedimento realizado (artroplastia X osteossíntese), a localização da lesão (MMII X MMSS) e o sítio cirúrgico específico.

\section{Análise estatística}

Os dados foram avaliados com auxílio do software SPSS ${ }^{\circledR} 12.0$ (Chicago, EUA). A análise univariada foi realizada utilizando o qui-quadrado $\left(\chi^{2}\right)$ para variáveis qualitativas ou o teste exato de Fisher quando uma das frequências era menor que cinco. Para variáveis quantitativas foi utilizada a análise de variância (ANOVA) tomando como significativos valores de $p<0,05$.

\section{RESULTADOS}

No momento do estudo $33(51,6 \%)$ pacientes haviam evoluído para óbito. Dos 64 pacientes submetidos a tratamento cirúrgico para metástases ósseas, 17 (26,6\%) apresentaram alguma complicação precoce no tratamento; seis $(35,2 \%)$ evoluíram para óbito em decorrência dessa complicação. As complicações cirúrgicas foram observadas em dez (15,6\%) pacientes, as complicações clínicas em quatro $(6,3 \%)$ e três casos $(4,7 \%)$ apresentaram tanto complicações clínicas quanto cirúrgicas. Dos pacientes que evoluíram com alguma complicação, quatro (23,5\%), necessitaram de um novo procedimento operatório. 
As complicações clínicas observadas foram trombose venosa profunda $(\mathrm{n}=3)$, tromboembolismo pulmonar $(\mathrm{n}=1)$, infarto agudo do miocárdio $(\mathrm{n}=1)$ e pneumonia $(\mathrm{n}=2)$. Quanto às complicações cirúrgicas, a mais frequente foi a hemorragia $(\mathrm{n}=8)$, infecções superficiais $(\mathrm{n}=2)$, infecção profunda $(\mathrm{n}=1)$, luxação da prótese $(\mathrm{n}=1)$, quebra da osteossíntese $(\mathrm{n}=1)$ (Figura 1).

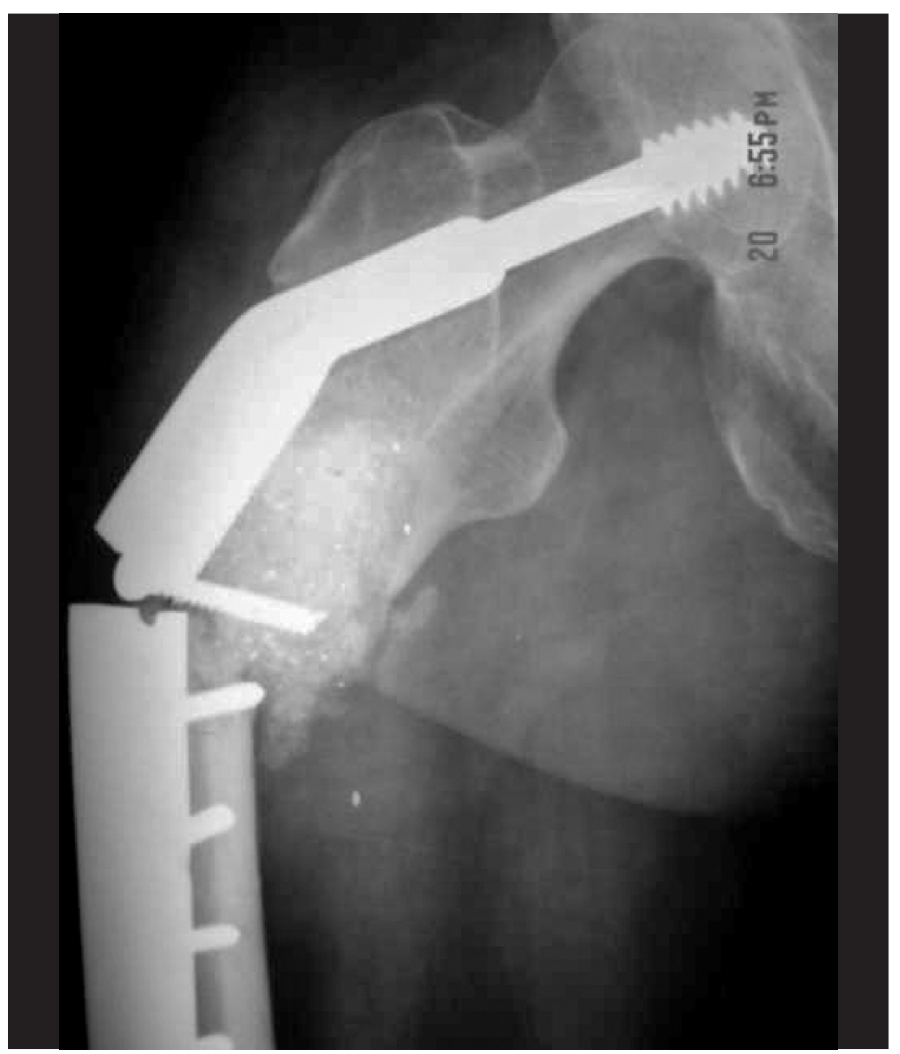

Figura 1 - Complicação pós-operatória caracterizada por quebra da osteossíntese e refratura após três semanas da cirurgia

Quando comparados com o tipo de procedimento cirúrgico, cinco $(20,8 \%)$ pacientes submetidos a artroplastia e $11(30,5 \%)$ a osteossíntese apresentaram alguma complicação, não sendo a diferença significativa $(\mathrm{p}=0,404)$. Quanto à mortalidade, dois $(8,3 \%)$ pacientes submetidos a artroplastias e três $(8,3 \%)$ a osteossíntese evoluíram para óbito, não sendo a diferença significante $(\mathrm{p}=1,000)$.

A média de reposição de unidades de hemácias foi de 1,54 unidade no grupo submetido a artroplastias e de 1,44 unidade no de osteossíntese, sendo a diferença não significativa $(\mathrm{p}=0,759)$. Quando avaliada em relação ao tumor primário, os tumores de origem renal apresentaram risco elevado de reposição de hemácias no peroperatório quando comparado com os outros tumores $(\mathrm{p}=0,0001)$.
Quanto à localização da lesão no esqueleto, não foram observadas diferenças significantes; os procedimentos cirúrgicos realizados nos MMII apresentaram 16 (27,1\%) complicações, sendo observadas em um (20\%) paciente lesões em MMSS ( $p=0,890)$. Quando avaliado cada sítio operatório específico, apesar da tendência de maior frequência de complicações na região subtrocanteriana, também não houve diferença significante $(\mathrm{p}=0,079)$.

\section{DISCUSSÃO}

O tratamento ortopédico objetiva o controle da dor, a estabilização de fraturas iminentes ou patológicas, manter a mobilidade, a marcha e evitar as complicações do decúbito prolongado ${ }^{(1,3-5)}$.

As opções de tratamento cirúrgico incluem próteses convencionais, endopróteses, osteossíntese simples ou associada ao uso de polimetilmetacrilato (cimento ósseo) e, mais raramente, amputação. A escolha do procedimento depende das condições dos pacientes, da extensão da doença e do local envolvido ${ }^{(1,4,5)}$.

Complicações peroperatórias são mais frequentes nesses pacientes, usualmente debilitados, desnutridos, com distúrbios metabólicos e hematológicos ${ }^{(1,6)}$.

Harrington et $a l^{(2)}$ analisaram 375 fraturas patológicas por MO e observaram como complicações: 13 infecções, 11 complicações tromboembólicas, dois casos de coagulação intravascular disseminada e um caso de disseminação metastática no sítio cirúrgico.

Park et $a l^{(7)}$ observaram, em 58 pacientes submetidos a tratamento cirúrgico de $\mathrm{MO}$ com artroplastias, ocorrência de infecção em $8,6 \%$ dos casos e de luxações em $12,9 \%$ nos primeiros dois meses de pós-operatório.

Thai et $a l^{(8)}$, avaliando 96 cirurgias para MO no úmero, encontraram como complicação mais comum a paralisia do nervo radial em $6 \%$ dos casos e como complicação clínica o tromboembolismo pulmonar em dois casos.

Em nosso estudo observamos incidência relativamente alta de complicações $(26,6 \%)$ compatível com a maioria das casuísticas estudadas ${ }^{(3-6)}$, sendo as complicações cirúrgicas mais comuns, em especial a hemorragia. A ocorrência de sangramento aumentado deve-se à hipervascularização, efeitos sistêmicos do tumor e do tratamento quimioterápico e radioterápico ${ }^{(1,5)}$. Os tumores de origem renal apresentaram risco importante de hemorragias e necessidade de reposição sanguínea maior que os de outros tumores; isso sugere a necessidade de embolizações pré-operatórias de rotina. 
As infecções ocorreram em 4,8\% dos casos, compatível com outros trabalhos da literatura ${ }^{(4-10)}$. Jacofsky et $a l^{(11)}$, avaliando 42 casos de artroplastias de quadril para tratamento de tumores malignos primários e metastáticos, encontraram como complicação mais comum a infecção profunda, com incidência de quase $10 \%$ da casuística.

Falência mecânica da reconstrução foi observada em um caso de osteossíntese de uma fratura subtrocanteriana. No fêmur proximal, por ser região de grande estresse mecânico, vários autores sugerem que a reconstrução deva ser feita com próteses não convencionais, evitando complicações decorrentes da indicação da osteossíntese $^{(7,10,11)}$, como a observada na figura 1 .

Do ponto de vista clínico, a complicação mais frequente foi o tromboembolismo, que ocorreu em 7,7\% dos casos, sendo um de embolia pulmonar maciça resultando em óbito no pós-operatório imediato. Esses achados são semelhantes aos observados por Marco et $a l^{(9)}$, que relataram a trombose venosa profunda e as infecções superficiais como responsáveis por $67 \%$ das complicações precoces do tratamento cirúrgico de MO do acetábulo. Nathan et $a l^{(12)}$ sugerem que nesses pacientes a profilaxia farmacológica deva ser complementada por uso de métodos mecânicos, pelo risco elevado de trombose venosa.

Em nossa casuística, as complicações foram frequentes e resultaram em $35,2 \%$ de óbitos e $23,5 \%$ de novas intervenções cirúrgicas; isso sugere que são usualmente mais graves e com maior morbidade nesses pacientes.

A maioria dos estudos não compara as complicações precoces entre os diferentes procedimentos ortopédicos $^{(8-10)}$, sendo as avaliações realizadas do ponto de vista funcional e da falência tardia do método de fixação. Nosso estudo abordou apenas as complicações precoces, objetivando comparar o porte cirúrgico e suas consequências nos pacientes com doença metastática. Ao contrário de alguns trabalhos que sugerem menor índice de complicações no uso de artroplastias, quando comparado com o de osteossínteses ${ }^{(8-10)}$, nosso estudo não mostrou diferença na incidência de complicações, na mortalidade ou na necessidade de reposição sanguínea, sugerindo que ambos os procedimentos são compatíveis do ponto de vista das complicações peroperatórias.

\section{CONCLUSÃO}

A incidência de complicações associadas ao tratamento ortopédico das MO foi de $26,6 \%$, com mortalidade de $35,2 \%$, sendo as complicações mais frequentes as hemorragias, a infecção e os eventos tromboembólicos. As complicações não dependem do tipo de procedimento ou do local acometido. Os tumores de origem renal apresentaram risco maior de sangramento e hemorragias.

\section{REFERÊNCIAS}

1. Finn HA. General considerations. In: Simon MA, Springfield D, editors. Surgery for bone and soft tissue tumors. Philadelphia: Lippincott-Raven, 1998;609-13.

2. Harrington KD, Sim FH, Enis JE, Johnston JO, Diok HM, Gristina AG. Methylmethacrylate as an adjuvanct in internal fixation of pathological fractures: experience with three hundred and seventy-five cases. J Bone and Joint Surg Am. 1976;58(8):1047-55.

3. British Orthopaedic Association and the British Orthopaedic Oncology Society. Metastatic bone disease: a guide to good practice; 2001.

4. Healey JH, Brown HK. Complications of bone metastases: surgical management. Cancer 2000;88(12 Suppl):2940-51.

5. Capanna R, Campanacci D. The treatment of metastases in the appendicular skeleton. J Bone Joint Surg Br. 2001;83(4):471-81.

6. Bibbo C, Patel DV, Benevenia J. Perioperative considerations in patients with metastatic bone disease. Orthop Clin North Am. 2000;31(4):577-93.

7. Park DH, Jaiswal PK, Al-Hakin W, Aston WJS, Pollock RC, Skinner JA, et al. The use of massive endoprosthesis for the treatment of bone metastases. Sarcoma. 2007;2007:62151.
8. Thai DM, Kitagawa Y, Choong PFM. Outcome of surgical management of bony metastases to the humerus and shoulder girdle: a retrospective analysis of 93 patients. Intern Semin Surg Oncol. 2006;3:5. Available from: www.isoonline.com/ content/3/1/5 - doi:10.1186/1477-7800-3-5

9. Marco RA, Sheth DS, Boland PJ, Wunder JS, Siegel JA, Healey JH. Functional and oncological outcome of acetabular reconstruction for the treatment of metastatic disease. J Bone Joint Surg Am. 2000,82(5):642-51.

10. Josin P, Dutka J. Clinical and radiological evaluation of mechanical sufficiency of the operative treatment of pathological fractures in bone metastases. Orthop Traumatol Rehabil. 2003;5(3):290-6.

11. Jacofsky DJ, Haidukewych GJ, Zhang H, Sim F. Complications and results of arthroplasty for salvage of failed treatment of malignant pathologic fractures of the hip. Clin Orthop Relat Res. 2004;(427):52-6.

12. Nathan SS, Simmons KA, Lin PP, Hann LE, Morris CD, Athanasian EA, et al. Proximal deep vein venous thrombosis after hip replacement for oncologic indications. J Bone Joint Surg Am. 2006;88(5):1066-70. 